\title{
Os Programas Especiais e o Novo Modelo Assistencial
}

\author{
Maria da Glória Lima Cruz Teixeira* \\ Jairnilson Silva Paim**
}

\begin{abstract}
"Profa. Adjunto do Departamento de Medicina da Faculdade de Medicina da Universidade Federal da Bahia. Ex-Gerente da Vigilancia Epidemiologica da Secretaria da Salde do Estado da Bahia.
\end{abstract}

**Prof. Adjunto do Departamento de Medicina Preventiva da Faculdade de Medicina da Universidade Federal da Bahia. Ex-Assessor Especial da Secretaria da Salude do Estado da Bahia.
Os autores comentam a necessidade de traduzir para a organização dos serviços de saúde os princípios delineados pela VIII Conferência Nacional de Saúde. Reafirmam que o modelo assistencial proposto pelas Açôes Integradas de Saúde (AIS) e assumido pelo Sistema Unificado e Descentralizado de Saúde (SUDS) implica a modificação progressiva dos Programas Especiais de saúde pública. Descrevem os esforços empreendidos pela Secretaria da Saúde do Estado da Bahia, durante a implantação do SUDS, visando a compatibilização desses programas com o novo modelo assistencial. Analisam as resistências observadas, as tentativas de superação, e a transição (não concluida) do modelo assistencial e propōem um esquema de organização interna das unidades de saúde que contemple a demanda espontânea e a oferta organizada para problemas prioritários. Concluem que certos Programas Especiais podem continuar existindo por força do seu conteúdo técnico-científico desde que se adaptem às diretrizes politicas e aos princípios da Reforma Sanitária.

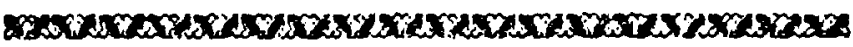

\section{INTRODUÇĀO}

Durante os três anos que se sucederam à realização da VIII Conferência Nacional de Saúde (2), desencadeou-se, no Brasil, um processo de discussão sobre a forma de traduzir para a organização dos serviços de saúde os princípios delineados no relatório daquela conferência.

Neste sentido, a implementação da estratégia das Ações Integradas de Saúde (AIS) estimulou a explicitação de um modelo assistencial que buscava abandonar a forma de organização dos serviços por programas verticais, horizontalizando-os pela destruição de linhas de mando e pelo fim dos recursos vinculados (7), mas com o cuidado para que as atividades da oferta organizada dos programas de saúde pública 
não sejam substituídas pelo mero atendimento à demanda espontânea (7).

Em alguns estados brasileiros a criação do Convênio SUDS (5) a fim de aperfeiçoar qualitativamente a estratégia das Açōes Integradas de Saúde (9) possibilitou que as Secretarias Estaduais fossem um pouco além do debate e que algumas mudanças concretas pudessem ser implementadas não só no âmbito políticogerencial como também no da organização de serviços (11).

A perspectiva de implantação do Sistema Único de Saúde, por força constitucional, torna necessária a reflexão sobre experiências vividas neste campo nos últimos anos. Neste sentido faz-se pertinente a discussão sobre a forma de inserção dos chamados Programas Especiais no Sistema Único de Saúde. No presente estudo utiliza-se a noção de Programa explicitada por Testa (14): conjunto de recursos, preferencialmente comandados por uma chefia administrativa única, destinados a alcançar um objetivo claramente definido. $\mathrm{O}$ seu caráter especial é assumido pela prioridade atribuída a grupos populacionais ou agravos específicos tais como: criança, mulher, idoso, tuberculose, hanseníase, doenças crônico-degenerativas, endemias etc. O Ministério da Saúde reconhece-os como objeto de ações especiais e programaticamente individualizadas (1) ao ponto de manter, na sua estrutura organizacional, até recentemente, uma Secretaria Nacional de Programas Especiais (SNEPS).

Um dos princípios de Reforma Sanitária é o da integralidade da atenção, em contraposição à assistência feita de forma fragmentada por agravo, aparelho ou sintomas, sem uma visão global do organismo humano e muito menos da inserção social do indivíduo ou dos grupos populacionais a que ele pertence. Conseqüentemente, faz-se pertinente analisar, no processo de construção do Sistema Único de Saúde (6), os princípios da organização da rede de serviços de saúde (3) à luz de cada experiência concreta. No caso da evolução dos Programas Especiais, por exemplo, há que se considerar, principalmente, a relação conflitante, à primeira vista, entre os princípios do impacto e o da não-rejeição da demanda (3): o primeiro reconhece que todo serviço é organizado com o objetivo de produzir mudanças no quadro ambiental e epidemiológico e não apenas para a produção de atividades preventivas elou assistenciais (3); já o segundo assinala que quando o primeiro nivel de complexidade rejeita pacientes, estes irâo à unidnde de maior complexidade, produzindo um exagerado uso dos serviços emergenciais, jă que a atenção dos pacientes, em última instância, é responsabilidade da rede de serviços e não só das unidades de saúde individualmente (3). 
$O$ presente artigo visa sistematizar os esforços empreendidos pela Secretaria da Saúde do Estado da Bahia, durante a implantação do SUDS, ao buscar a compatibilização dos chamados Programas Especiais com o modelo assistencial proposto a partir do princípio da integralidade da atençấo.

\section{OS PROGRAMAS ESPECIAIS NA SECRETARIA DA SAUDE DO ESTADO LA BAHIA}

$\mathrm{Na}$ Bahia, durante o período em que houve a decisão política de iniciar o processo de implantação da Reforma Sanitária no Estado (biênio 87-89), a discussão em tomo do que fazer com os programas existentes, e de como transformar as práticas já cristalizadas nos serviços de saúde na nova concepção da "atençāo integral", foi bastante profícua. Conseguiuse delinear algumas estratégias de atuação no sentido da construção da nova proposta de assistência à população. As diferentes posições dos dirigentes e corpo técnico da Secretaria da Saúde do Estado da Bahia (SESAB) iam desde aqueles que defendiam a necessidade de extinção imediata dos Programas Especiais, até aqueles outros que se debatiam para demonstrar, através de dados epidemiológicos ditos de impacto, a eficácia destes na rede de saúde, lutando pela sua conservação.

Estes programas estavam, até aquele momento, estruturados na Administração Central da Sesab através de Coordenações cujos Coordenadores encontravam-se isolados do corpo da assistência. Já, nas unidades de saúde, dispunham de responsáveis e especialistas para cada um deles, evidenciando uma excessiva verticalização. Ademais, as respostas administrativas e epidemiológicas de cada programa atendiam apenas aos interesses do organismo financiador ou instituição que o tinha proposto (ou imposto?) à Secretaria. Configuravam uma prática de gestão denominada "administração convenial". Mendes (8), analisando este modelo de planejamento adotado no âmbito nacional pelo regime autoritário, reconhece-o como expressấo de um financiamento público para a saúde cujos recursos eram provenientes do Governo Federal $(61,3 \%)$, dos Governos Estaduais $(28,5 \%)$ e dos Governos Municipais $(10,2 \%)$. Este "paroxismo centralista" criava uma situação na qual os recursos concentrados, com prioridades e restriçóes impostas segundo os interesses da União, pela tecnoburocracia federal, deveriam ser identificados e negociados pelos Estados e Municipios, através de convênios (8).

Conseqüentemente, os programas tinham seus próprios instrumentos de coleta de dados, programação 
elaborada separadamente e em épocas diferentes do ano, metas que variavam desde a erradicaçāo até a simples prestaçāo de assistência, independente do perfil epidemiológico do Estado, Região ou Município.

Aos Coordenadores destes programas competia não só implantar, implementar, avaliar, enviar dados em tempo e hora marcados pelas instituiçōes financiadoras e prestar contas do ocorrido no Estado, como também, e talvez principalmente, sensibilizar com seu esforço pessoal os dirigentes da Sesab para a importância do seu programa e, assim, garantir espaço para execução das atividades previstas na programação. $\mathrm{Na}$ prática cada programa tentava influenciar, via coordenador, as diretrizes da política de saúde da Secretaria de Estado. Todos estes pontos demonstravam que a existência deste sistema era claramente antagônico à proposta de uma atenção integral. Entretanto, cumpre reconhecer que as açöes realizadas na rede, sob a forma de Programa Especial, apresentavam um melhor desempenho do ponto de vista administrativo como também na qualidade da assistência prestada, não só por terem instrumentos de avaliaçāo bem definidos e recursos alocados, mas principalmente por se atribuírem responsabilidades a cada nivel institucional. Quanto aos outros agravos atendidos na rede, porém contemplados pelos Programas Especiais, além de nāo terem instrumentos de avaliação (exceto a simples contabilização do número de consultas e internamentos), não havia cobrança de responsabilidades mínimas aos profissionais ou dirigentes.

OS PROGRAMAS ESPECIAIS E AS PROPOSTAS DE REFORMA EM SAÚDE NA BAHIA

A necessidade de mudança da forma e da organização dos Progranıs Especiais ficava patente. Diversas questões relativas ao princípio da integralidade da atenção à saúde, já sistematizadas por Mendes (8) desde as AIS, mantinham-se pertinentes durante a implantação do SUDS, particularmente na organização dos Distritos Sanitários:

a) os programas convencionais de "Saúde Pública" deverão ser horizontalizados?

b) quais, por que e como?

c) a atenção médica deverá ser incorporada às unidades de saúde tradicionalmente prestadoras de serviços de oferta organizada?

d) por que e como?

e) as atividades de "saúde pública" deverāo ser incorporadas às unidades tradicionalmente ofertadoras de atençāo médica à demanda espontânea?

Cadernos de Saúde Pública, RJ, 6 (3): 264-277, jul/set, 1990 
f) por que e como?

g) como compatibilizar numa única unidade as atividades de atenção médica $e$ de saúde pública?

h) que conceito de resolutividade será adotado?

i) como evitar a possibilidade - dada pela autopercepção da clientela - de valorização desproporcional do componente da atenção à demanda espontânea em detrimento daquela da oferta organizada?

j) que modificações nas composições tecnológicas das unidades de saúde existentes serāo necessárias para haver resolutividade?

k) que modificaçōes essas novas composiçōes tecnológicas determinarão no planejamento físico das unidades de saúde?

l) que fazer com as atuais unidades que não atendem às novas exigências, no campo fisico?

$m$ ) será viável estabelecerem-se as atividades a serem ofertadas pela rede de serviços com base em critérios epidemiológico-sociais? (8).

Evidentemente que não se dispunham, desde 0 início, de respostas para todas estas questōes. Mas reconhecia-se que a transformação do modelo assistencial teria de ser feita, porém sem agravar a situação sanitária e administrativa, pois não se pretendia criar qualquer soluçāo de continuidade na assistência oferecida na rede. Isto porque a demanda espontânea por atenção à saúde é contínua e não pode aguardar a elaboração do desenho ideal, nem o tempo necessário para a implantação deste novo sistema (13). Ela precisava ser atendida enquanto se introduziam mudanças no modelo assistencial.

$\mathrm{Na}$ Figura I, procura-se ilustrar o modelo inicial em que há o predomínio dos Programas Especiais e da demanda espontânea e o novo modelo assistencial, em que os Programas Especiais são substituídos progressivamente pela oferta organizada (por grupos populacionais e/ou agravos) mantendo, no interior da unidade de saúde, relações funcionais e programáticas com a demanda espontânea, relativamente reduzida.

Apesar da postura hegemônica no debate inicial ter dado uma ênfase muito grande na necessidade da extinção dos Programas Especiais, por eles simbolizarem a "prática vertical", optou-se por uma mudança gradativa como estratégia de transição (13), para a construção de um sistema mais horizontal de atenção integral à população.

As modificaçōes foram iniciadas pelo Nivel Central, nas áreas de Vigilância Epidemiológica, Tuberculose, Hanseníase, Raiva, Imunização etc, com os coordenadores socializando as informações sobre conteúdo, metas, atividades, dados epidemiológicos e situação dos programas para todo o corpo técnico da Coorde- 
nação onde estes estavam inseridos. As informações, dependendo do tipo de agravo, passaram a ser periodicamente computadas pelos profissionais envolvidos e imediatamente repassadas ao corpo técnico e dirigentes da Secretaria, enquanto o Centro de Informaçôes de Saúde (CIS) ia se reestruturando e informatizando para trabalhar com dados epidemiológicos.

\section{FIGURA I}

Diagrama de Transição dos Programas Especiais para a Oferta Organizada do Novo Modelo Assistencial

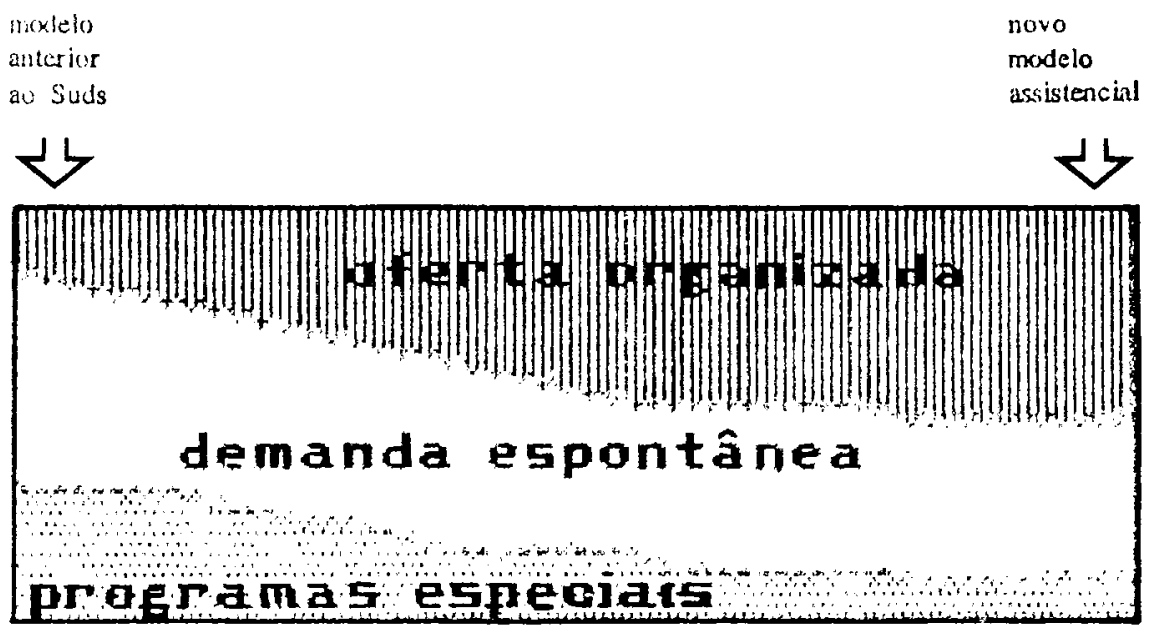

O Nível Regional foi orientado no sentido de proceder da mesma forma que o Nivel Central. Já no Nivel Local, as mudanças solicitadas foram mais complexas, visto que implicavam a reorganização das unidades de saúde e a transformação da prática cotidiana dos profissionais na sua relação com os pacientes. Logo de início, propôs-se que os médicos dos Programas Especiais também atendessem pacientes com outras patologias, ou seja, que fizessem clínica médica, e que os clinicos atendessem pacientes com patologias pré-definidas, o que historicamente só era feito pelos especialistas, a exemplo de tuberculose, hanseníase etc. Além disso, nos Centros de Saúde, optou-se pela não-existência de enfermagem ou assistência social especializada por agravo.

Acentuou-se a exigência no preenchimento dos prontuários já implantados na rede, observando-se uma 
rotina mínima com anotações de anamnese, exame físico, suspeita clínica, plano diagnóstico e plano terapêutico, independente da patologia do paciente ou do serviço da unidade. Pretendia-se que o paciente fosse visto como um todo e não segmentado, como nos casos de portadores de Tuberculose Pulmonar e Hanseníase, que só tinham seu pulmão ou a pele e sistema neurológico periférico examinados. Este item da proposta tinha uma aceitação a nível do discurso da maioria dos profissionais. Entretanto, não havia uma disposição evidente de mudança da prática de rotina, na busca de uma melhor qualidade da atenção, nem um comprometimento por parte da direção das unidades de saúde no sentido da sua reorganização interna no âmbito dos serviços de triagem, dos fluxos, das rotinas etc, para se adequarem ao novo modelo assistencial.

\section{RESISTÊNCIAS AO MODELO ASSISTENCIAL E TENTATIVAS DE SUPERAÇĀO}

Frente a estas propostas, observou-se imediatamente uma grande reação dos profissionais. No Nível Central esta reação pôde ser melhor contomada, porque os Coordenadores foram mantidos como "especialistas dos agravos", respondendo pelas ações junto ao Ministério da Saúde, mas a programação, viagens, repasse de dados, treinamentos etc, eram feitos em consonância com a nova diretriz política imprimida à Secretaria. As decisões passaram a ser do conhecimento e com a anuência dos Chefes imediatos e nunca tomadas apenas numa negociação direta entre Coordenador e agência financiadora. A programação da Secretaria para o ano de 1988 (POI-88) (12) foi elaborada a partir de oficinas de trabalho realizadas por Municípios e Diretorias Regionais, com a participação das formas organizadas da sociedade civil. Assim, os Programas Especiais tiveram que contemplar as necessidades apontadas pelo Nível Local, e, a partir destas, fazer as solicitaçóes às agências financiadoras, obtendo-se, a nível de programação, a inversão da forma como este processo anteriormente se desenvolvia.

No Nível Local, a reação dos profissionais, particularmente dos médicos, foi muito mais intensa, gerando grandes dificuldades para o gerenciamento das Unidades de Saúde. Nas áreas de implantação dos Distritos Sanitários, onde havia por parte da Administração Central uma atenção especial, estas dificuldades foram parcialmente contomadas, com discussões e esclarecimentos constantes junto aos profissionais das Unidades de Saúde e com a realização concomitante de cursos e treinamentos para capacitação técnica das equipes, dentro da perspectiva do novo modelo assistencial. Observou-se, naquele momento, que a reação à nova 
proposta se dava não só devido ao conteúdo do trabalho médico, mas que este reclamo servia principalmente como pretexto para extravasar a reação a outras exigências da nova administração: cumprimento da carga horária contratual e o controle do tempo mínimo dispensado para cada paciente atendido.

Como forma de discutir, informar e obter subsidios para o melhor desempenho da assistência, foram realizadas avaliaçōes periódicas gerais e setoriais, com a apresentação de dados e análises epjdemiológicas. Destas avaliaçōes participavam representantes das Diretorias Regionais, dos Distritos Sanitários já implantados e dirigentes do Nivel Central. Através destas, traçavam-se estratégias de atenção a curto prazo, principalmente nas áreas de imunização, vigilância epidemiológica e sanitária, objetivando corrigir as falhas e executar ações que provocassem algum impacto no perfil epidemiológico de cada regiảo ou município.

Durante o desenvolvimento desse processo, foram também analisados os pontos positivos existentes nos Programas Especiais, que pudessem contribuir para a construção do novo modelo assistencial. Observou-se que tais programas possuíam instrumentos de avaliaçâo, na maioria das vezes bem elaborados, com indicadores definidos, indispensáveis ao controle de qualidade e do desempenho das açōes, desde o exame clínico, passando por critérios diagnósticos e padronização terapêutica, elementos indispensáveis ao controle de qualidade e desempenho das açöes. Este foi um ponto destacado, por servir como parâmetro na elaboração de normas e rotinas de serviços. Os mecanismos de gerenciamento dos programas, apesar de necessitarem de uma revisão crítica pela excessiva verticalização, apresentavam pontos positivos que podiam ser resgatados para aplicação no novo modelo de assistência.

\section{A TRANSIÇÃO (NĀO CONCLUÍDA) PARA O NOVO MODELO ASSISTENCIAL}

Considerando-se que o problema do financiamento do setor saúde pode não estar resolvido em um curto intervalo de tempo, a captação de recursos através de Programas Especiais junto a agências financiadoras não-governamentais e intemacionais pode constituir-se em um caminho ainda a ser trilhado. Deve-se, entretanto, negociar com as agências a definição de prioridades de implantação e operacionalização além da alocação de recursos, tendo como referencial a sitvação de saúde (perfil epidemiológico da população e características dos serviços locais). 
Ademais, o conteúdo técnico-científico dos Programas Especiais tem grande importância para a garantia da qualidade da assistência e não deve ser subestimado, inclusive pelo fato de os recursos humanos existentes na rede nao terem condições de assumir a totalidade das atividades desses programas, sem uma prévia capacitação geral e específica. Tal conteúdo é fundamental para a eficácia e as especificidades das açōes necessárias ao controle de determinados agravos, mas não deve ser instrumentalizado como expediente para determinar a forma de organização dos serviços.

Diante da análise desses aspectos, observa-se que o novo modelo de assistência integral requer uma fase de transição, dependendo do grau de desenvolvimento das Unidades de Saúde e dos mecanismos de discussão, repasse e supervisão programados (13). Provavelmente este novo modelo será híbrido, isto é, organizado por faixa etária e sexo (atenção à criança, à mulher, ao idoso etc), atendendo à demanda espontânea e também à oferta organizada. A partir das suspeitas ou diagnósticos formulados, os problemas poderäo ser resolvidos nesse nível de atendimento ou referidos para serviços especializados por agravos. Para isso, faz-se necessária a elaboração e/ou revisão de normas e rotinas de atendimentos em todos os níveis, particularmente no âmbito da Unidade de Saúde. A revisão dos papéis e atribuições dos profissionais está indicada, pois, mesmo que um determinado agravo pertença a um Programa Especial, o diagnóstico e tratamento poderão ser efetuados pelo médico generalista e só serão referidas para os especialistas aquelas situaçōes mais complexas, previamente normatizadas, que necessitem de uma atenção especial. Esta oferta organizada de atendimento é necessária para doenças infecciosas e parasitárias, crônico-degenerativas, ou aquelas que necessitem de investigação para esclarecimento diagnóstico.

A Figura II apresenta uma adaptação do esquema proposto por Capote (4), contemplando o conjunto de elementos para análise do enfoque por problemas. Sugere alguns fluxos que permitam compatibilizar, no interior da unidade, a demanda espontânea com a oferta organizada, dentro do princípio da integridade da atenção. Nota-se que o impulso dominante para a demanda espontânea busca consultas, pronto-atendimento ou mesmo atenção de urgência e emergência. A unidade de saúde precisa ter um Same organizado, triagem normatizada e um serviço de pronto-atendimento capaz de atender o paciente ou encaminhá-lo devidamente, através do sistema de referência-contrareferência (RCR). No caso do atendimento na unidade, a consulta seria efetuada para grupos de populações (10) (criança, mulher em idade fértil, adulto em geral, 
idoso), cujos serviços disporiam das rotinas para identificação e controle dos agravos reconhecidos como problemas prioritários através de estudos epidemiológicos. A "oferta organizada" das açōes voltadas para tais problemas incluiria não apenas o conjunto de pacientes captados pela demanda espontânea, mas também a busca ativa de casos e o respectivo controle no âmbito comunitário ("dispensarizaçāo"). Assim, a unidade de saúde, organizada de acordo com o princípio da integralização da atenção, articularia o conjunto das ações de saúde (promoçăo, proteção, recuperação e reabilitação) sobre individuos e sobre o ambiente, visando o controle de agravos (TRO, IRA, Imunizaçōes etc) e a proteção de grupos vulneráveis. Isto supõe que todo e qualquer contato dos usuários e seus familiares com a unidade de saúde (mesmo que em busca de um simples atendimento médico), bem como as visitas domiciliares e as relações entre os serviços de saúde e as entidades e equipamentos comunitários (associaçōes de moradores, igrejas, escolas, creches etc) deverão ser utilizados com vistas à promoção, proteção e recuperação da saúde, fundamentando-se nas normas específicas da oferta organizada. Assim, uma gestante que leva uma criança para o atendimento em pediatria deve ser convidada a inscrever-se no serviço de pré-natal; um homem adul to que acompanha a mulher para uma consulta ao ginecologista pode ter tomada a sua pressâo arterial e ser alertado para as conseqüências do hábito de fumar.

A organização intema das unidades bem como as normas e rotinas elaboradas deverão dar conta não só da atenção médica individual, mas também das ações coletivas a serem adotadas para cada situação diagnosticada. Os profissionais da Unidade de Saúde deverão ter conhecímento das ações a serem adotadas, se foram ou não realizadas pelo setor responsável, acompanhar o desenvolvimento destas açōes não só dos seus pacientes, mas os da sua unidade. Para isso, é importante que haja mecanismos coletivos de avaliação das Unidades e dos profissionais e ainda o acompanhamento do perfil epidemiológico da comunidade circunscrita àquela Unidade de Saúde. No caso da Bahia, ainda são poucas as iniciativas que avançaram neste sentido, o que deixa margem a um retrocesso do que foi construído. Todavia a manutençāo, ainda que inercial, dos Distritos Sanitários permite um campo de experimentação e desenvolvimento no que se refere à organizaçẩo das unidades de saúde. 


\section{FIGURA II}

Articulação da "Demanda Espontânea" com a "Oferta Organizada" no Âmbito da Unidade Local de Saúde.

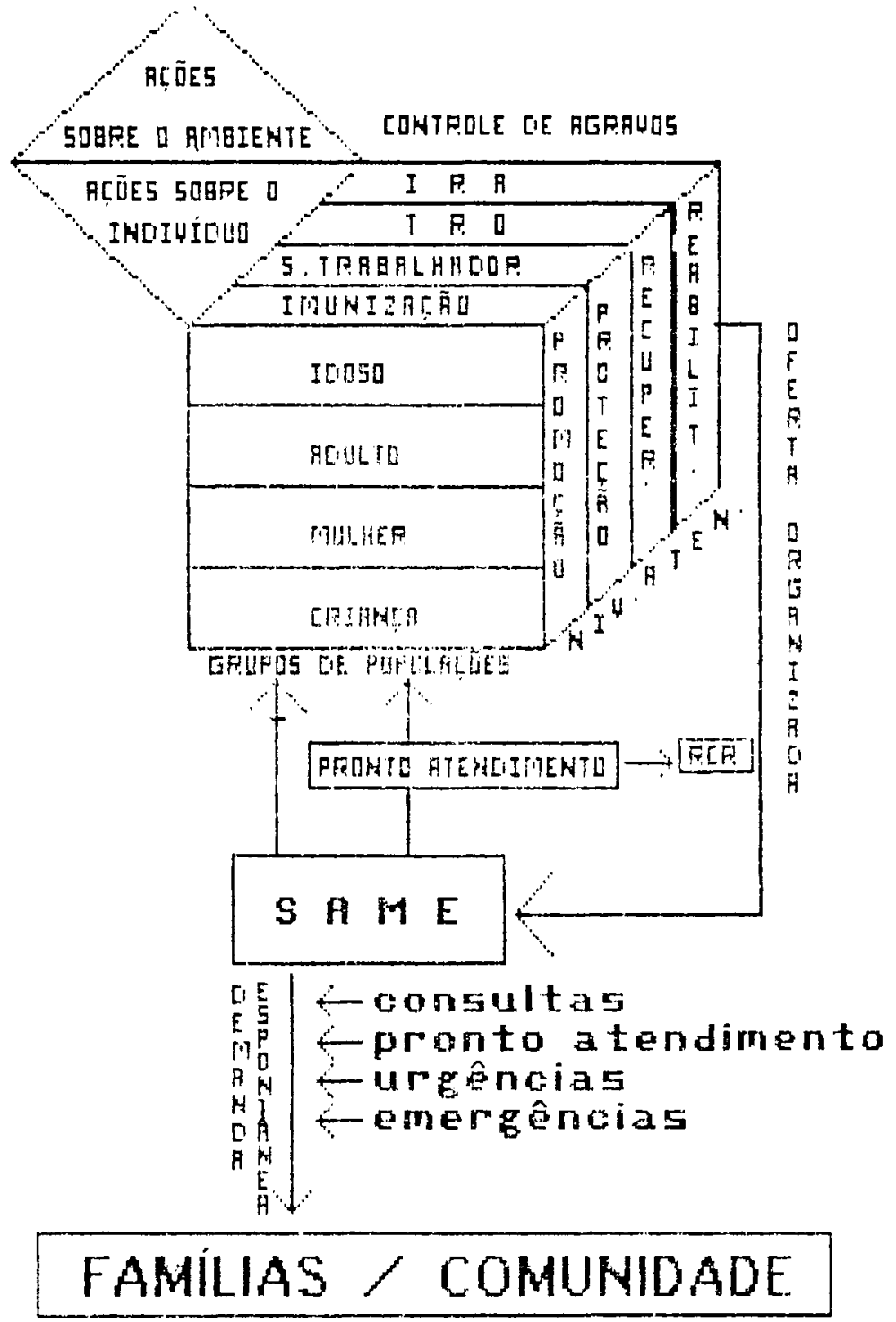




\section{COMENTÁRIOS FINAIS}

A experiência da Bahia com os Programas Especiais, durante o periodo de 87-89, em grande parte na vigência do Convênio SUDS, aponta para as seguintes reflexōes:

1. Desde que a adaptem às diretrizes políticas e princípios da Reforma Sanitária, certos Programas Especiais podem continuar existindo por força da complexidade do seu conteúdo técnico-científico, enquanto outros serăo absorvidos pela assistência organizada por grupamento populacional no decorrer do processo de implantação do novo modelo de assistência. Desta forma, alguns Programas Especiais continuarão tendo um, importante papel no desenvolvimento do Sistema Único de Saúde (SUS).

2. $\mathrm{Na}$ medida em que outras áreas de atuação sejam absorvidas pelo SUS, novos Programas Especiais poderäo ser criados para atender aos requisitos do conteúdo específico e/ou na fase inicial de implantação e capacitação da rede de saúde. Para ilustrar, podemos citar como exemplos: Saúde do Trabalhador, pelo nível de complexidade não só no campo diagnóstico e terapêutico, como também pelas repercussōes trabalhistas e relações com a Vigilância Epidemiológica e Sanitária; a AICS, por ser um agravo de diagnóstico recente; e situaçōes inusitadas que necessitem de medidas especiais de atenção.

3. Para fortalecer a proposta de implantação da Reforma Sanitária no Brasil, dirigentes e profissionais comprometidos com este processo devem ter uma preocupação constante em não criar situações que aumentem o sofrimento e risco para a população, mesmo numa perspectiva de avanço gerencial posterior. Assim, o processo de mudança deve ser no sentido de apresentar resultados que legitimem a proposta frente à população, garantindo-se assim o avanço da Reforma Sanitária. Desta forma estarão se criando condiçōes políticas necessárias aos enfrentamentos com os setores contrários à implantacão do SUS.

4. O SUS deve estabelecer mecanismos que garantam a incorporação dos avanços científicos e tecnológicos que atendam às necessidades de saúde da população. Estes avanços têm que ser criticamente analisados, para que não sejam incorporados de forma aleatória ou para atender interesses econômicos alheios às reais necessidades de atenção à saúde. Historicamente, os Programas Especiais têm experiência em sistematizar e adaptar os novos conhecimentos para a rede de serviços de saúde, sob várias formas. O resgate destas experiências, como é o 
caso da Terapia de Reidratação Oral (TRO) e do Programa de Controle da Tuberculose, contribuirá para o desenvolvimento do novo sistema.

5. Durante o processo de implantação e implementação das mudanças necessárias para se atingir 0 patamar delineado pela Reforma Sanitária, é preciso vigilância e debates constantes para que não se perpetue a tendência verticalizadora dos Programas Especiais. A estratégia a ser adotada deve se voltar para a instrumentalização dos pontos positivos destes programas, na perspectiva da horizontalização progressiva da organização da atenção à saúde.

Os autores registram os seus agradecimentos às Professoras Joselita Nunes Macedo e Vera Lúcia Formigli, do Departamento de Medicina Preventiva da UFBA, pelas valiosas sugestōes durante a discussão do presente artigo.

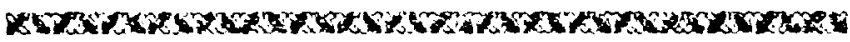

The authors comment on the need to translate into operational instructions the principles and concepts that guided the VIII National Conference in Health convened by the Ministry of Health of Brazil, to make possible the reorganization of the health system. It is emphasized that the model for organizing the primary health care proposed by the Integrated Health Actions (AIS) and assumed by the Unified and Decentralized Health System (SUDS), implies a progressive redefinition of the especial programs in public health. The authors describe the efforts of the Health

Department of the State of Bahia (SESAB) during the implementation of the SUDS to make compatible the operations of the especial programs with the primary health care model. The resistance observed to the new directions and the attempts to overcome it, are analysed, as well as the transition (unfinished) to the proposed health care model. It is proposed an alternative model for the internal organization of health care units which take into consideration the emerging demands and also the available offers to deal with priority health problems. As conclusion, the authors state that some special programs can continue to be developed within a unified and decentralized health care model, due to their technical and scientifical value, since these programs are adequated to the political principles of the Health System Reform in Brazil. 


\section{REFERÊNCIAS BIBLIOGRÁFICAS}

1. BRASIL. Conferéncia Nacional de Saúde, 7: Brasılia, 1980. Anais da 7: Conferencia Nacional de Saúde. Brasnia, Centro de Documentação do Ministério da Saúde, 1980, p. 235.

2. BRASIL. Conferência Nacional de Saúde, 8: Brasflia, 1986. Anais 8: CNS Brasilia 1986. Centro de Documentaçāo do Ministério da Saúde, 1987, p. 381-389.

3. CAPOTE MIR, $R$ * "O processo de organização e a regionalizaçåo dos serviços". In: São Paulo (Estado) Secretaria do Estado da Saúde. Instituto da Saúde. SILOS; Sistemas locais de saúde. Săo Paulo, Instituto de Saúde, 1988, p. 17-26.

4. CAPOTE MIR, R. Sistemas locais de saúde. Anotaçōes da exposição para técnicos e gerentes de distritos sanitários da Secretaria de Saúde do Estado da Bahia. Salvador, agosto de 1988.

5. CONVÊNIO 232/87. Dispōe sobre o Sistema Unificado e Descentralizado de Saúde do Estado da Bahia Diário Oficial da Uniäo: 139, 118838 de $14 / 7 / 87$.

6. GRUPO DE ASSESSORAMENTO PARA ELABORAÇĀO DA LEGISLAÇĀO SOBRE SEGURIDADE SOCIAL PÓS-CONSTITUINTE. Nácleo de Estudos em Saúde pública. Faculdade de Ciências da Saúde. Universidade de Brasilia. Anteprojeto de lei orgânica do Sistema Único de Saúde (proposta para discussão). Brasnia, fev. 1989, 51 p.

7. MENDES, E. V. Açös Integradas de Saúde e Modelo Assistencial. Brasflia, nov. 1985,11 p. (mimeo).

8. MENDES, E. V. Modelos assistenciais. (Texto preliminar) Brasîlia, mai. 198616 p. (mimeo).

9. MENDES, E. V. O Sistema Unificado e Descentralizado de Saúde no contexto da Reforma Sanitária. (Conferência proferida no Seminário Intemacional sobre Reforma Sanitária e a Organização dos serviços de saúde do Rio Orande do Norte). Set. 1987, 20 p. In: São Paulo (Estado) Secretaria de Saúde. Instituto de Saúde. SILOS Sistemas locais de saúde. São Paulo, Instituto de Saúde, 1988, p. 5-14.

10. MINISTÉRio DA SAÚDE. Secretaria Nacional de Açōes Básicas de Saúde. Divisåo Nacional de Organização de Serviços de Saúcle. Orientação para organizaçāo de Centros de Saíde. Brasfila, Centro de Documentaçāo do Ministério da Saúde, 1982. 27 p. (Série A: Norma e manuais técnicos).

11. PAIM, J. S. A Gestäo do SUDS no Estado da Bahia, Cadernos de Salide Pública. RJ, 5(4): 365-375, out/dez 1989.

12. SISTEMA UNIFICADO E DESCENTRA LIZADO DE SAÚDE DO ESTADO DA BAHIA. Programaçâo orçamentaçâo integrada (POU 88/89), jan. 1988, $225 \mathrm{p}$.

13. TEIXEIRA, M.G.L.C.; PAIM, J.S. Reorganizaçāo e desenvolvimento da Vigilância Epidemiologica na Perspectiva do Sistema Unificado e Descentralizado de Saúde (SUDS-BA) Salvador, 1989, 36 p. (mimeo).

14. TESTA, M. Planificación estratégica en el sector salud. CENDES/UNC, 1981, 20 p. (mimeo). 